\title{
Turbulent Inflow Noise Prediction from Wind Turbine Blades
}

\author{
Vasishta bhargava ${ }^{1}$, Jaya Krishna.M ${ }^{2}$, Dr Hima Bindu Venigalla ${ }^{3}$, YD Dwivedi ${ }^{4}$ \\ 1,2 Department of Mechanical Engineering, GITAM University, Hyderabad, Telangana, India \\ ${ }^{3,4}$ Department of Aeronautical Engineering, GITAM University, Hyderabad, Telangana, India
}

\begin{abstract}
In general, wind turbine blades operate in subsonic regions along with turbulent flow conditions. This paper deals with noise due to turbulent inflow around the blades based on Amiet's and Lowson's model formulation. This mechanism is predicted using numerical simulation for different observer azimuth positions and various rotor speeds of turbine. The influence of turbulence intensity, length scales on the sound pressure levels is illustrated. The effect of blade geometry characteristics on sound pressure levels is implemented. The influence on power production from wind turbine due to the reduction in sound power levels are correlated with the modified blade geometry. The total noise from turbulent boundary layer trailing edge and turbulent inflow mechanisms are compared for three turbines of sizes, $350 \mathrm{~kW}, 2 M W \& 3 M W$. Overall sound pressure levels are dominated by the inflow turbulence mechanism. The amplitude modulation is calculated using the sound pressure levels obtained and illustrated for predominantly low and medium range frequencies in octave spectra. Keywords: Turbulence intensity, Integral length scale, Sound pressure level, Wind Turbine, Airfoil.
\end{abstract}

\section{Introduction}

Turbulence phenomenon is caused due to various factors in atmosphere. The boundary layer characteristics and the surface roughness provide key information related to turbulent wind flows. They usually result in wind shear due to velocity gradients in boundary layer (Lee, et al). Temperature inversions affect the sound waves to undergo reflection refraction, while diffraction around obstacles cause formation of shadow zones (Hubbard, M. Friman). The extent of turbulence in the atmosphere can be measured using the turbulence intensity, length scale, time scale of operation, coherence functions that depict the size of eddies and energy content present in eddies.

Lighthill's acoustic analogy for far field sound pressure lays important significance in the study of broadband noise prediction for a source located in outdoor environment .It has been found from previous studies Lowson and Amiet (1979) that turbulent inflow noise is affected by the integral length scale and turbulence intensity levels. However, the studies showed that noise levels for low frequency range are predicted accurately using correction factors which are function of convective wave number, chord lengths of airfoil. The leading edge of airfoils interact with the turbulent eddies in wind during operation and produce noise levels which dominate over other noise mechanisms. Next sections will discuss about analytical formulations of acoustic pressure developed by several researchers, during 1980-84. The results are presented for the inflow turbulence phenomenon and compared for machine sizes of $350 \mathrm{~kW}, 2 \mathrm{MW}$ and $3 \mathrm{MW}$ which predict the sound pressure levels from non-stationary noise source, for blade lengths of order $17-47 \mathrm{~m}$ and airfoil chord lengths of $\sim 4 \mathrm{~m}$. Design of quieter turbine blades is governed by parameters tip speed, wind speed and rotor size dependent on blade span and atmospheric length scales. Noise produced from the trailing edge of blade contributes significantly and underlying physical mechanism responsible for trailing edge noise generation is beneficial in the design of quieter wind turbine blades and blades of an aircraft or helicopter $[1,8]$.

\subsection{Amiet's formulation}

\section{Model Description}

The earliest known work on acoustic field is governed by Lighthill (1954) [1] acoustic analogy containing the propagation and generation of sound terms. It does not take account noise generated from moving solid body surface and it consists of the stress tensor term on the RHS which indicates the transport of momentum in time and space by a fluctuating fluid $[19,20]$ that exhibits quad pole characteristic. The work was extended by Ffowcs Williams Hawkings [2] who developed the model for far field into generalized function consisting of additional terms on RHS and applied it for moving surface which utilizes the boundary layer characteristics. It includes the pressure term which represents the force which object exerts on fluid and exhibits a dipole like characteristic. The acceleration potential term represents the monopole characteristic of noise radiated from non stationary solid surface. The LHS term signifies the propagation of sound by the fluid flow while the RHS terms signify the generation of sound. The theoretical method for far field noise approximation as formulated by Amiet [1] accounts for incident pressure upstream of trailing edge of airfoil can be analyzed using two approaches 
a) Analytical representation for sound pressure level at different observer positions

b) Numerical simulation of noise propagation that includes geometric divergence

The pressure fluctuations travel as sound waves is known as acoustic pressure and function of time and space.

From the law of fundamental physics, the propagation speed of sound for an ideal gas is

$$
\mathrm{c}^{2}=\left(\frac{\partial \mathrm{p}}{\partial \mathrm{p}}\right)=\gamma \mathrm{RT}
$$

The acoustic pressure for pure tones can be expressed mathematically as

$$
\mathrm{p}^{\prime}(\mathrm{t}, \mathrm{x})=\mathrm{A} \cos \left[2 \pi\left(\frac{\mathrm{x}}{\mathrm{E}} \pm \frac{\mathrm{t}}{\mathrm{T}}\right)\right]
$$

Where, $\mathrm{A}$ - pressure amplitude, Tis the time period in $\mathrm{s}, \mathrm{C}$ is the wave length. The classical wave equation by Lighthill (1978) can be written as

$$
\begin{aligned}
& \frac{\partial^{2} p^{y}}{\partial t^{2}}-c_{0}^{2} \frac{\partial^{2} p^{y}}{\partial x_{i}^{2}}=\frac{\partial^{2} T_{i j}}{\partial x_{i} x_{i j}} \\
& \mathrm{~T}_{i j}=\partial u_{i} u_{j}-\tau_{i j}+p^{\prime}-c_{0}^{2} \rho^{\theta} \delta_{i j}
\end{aligned}
$$

Where, $\rho$ ' is the fluctuating fluid density $\rho-\rho_{0}, T_{i j}$ is the Lighthill stress tensor, $u_{i}$ is the flow velocity in I direction, $\tau_{\mathrm{ij}}$ Is the viscous force, p' is fluctuating fluid pressure, $\delta_{\mathrm{ij}}$ is the Kronecker's delta. The last three terms in eq (2) are ignored in the aero-acoustic prediction. Hence, the first term remains and also known as "Reynolds stress" tensor. However, Amiet extensions and Lowson corrections further developed the model with the trailing edge and inflow turbulent noise phenomenon after considering the low frequency correction factor and compressible sears function $\mathrm{S}$.

$$
\begin{aligned}
& \mathrm{SPL}_{\text {inflow }}=\mathrm{SPL}_{\text {inflow }}^{\mathrm{H}}+10 \log \left(\frac{\mathrm{LFC}}{1+\mathrm{LFC}}\right) \\
& \mathrm{SPL}_{\text {inflow }}^{\mathrm{H}}=10 \log \left(\frac{\mathrm{\rho}^{2} \mathrm{c}^{2} \mathrm{LL}}{2 \mathrm{r}_{2}^{2}} \mathrm{M}^{2} \mathrm{u}^{2} \mathrm{I}^{2} \frac{\mathrm{K}^{3}}{\left(1+\mathrm{K}^{2}\right)^{7 / 3}} \overline{\mathrm{D}}_{\mathrm{L}}\right)+58.4 \\
& \mathrm{LFC}=10 \mathrm{~S}^{2} \mathrm{MK}^{2} \beta^{-2} \\
& \mathrm{~S}^{2}=\left(\frac{2 \pi \mathrm{K}}{\beta^{2}}+\left(1+2.4 \frac{\mathrm{K}}{\beta^{2}}\right)^{-1}\right)^{-1} \text { where } \beta=\sqrt{1-\mathrm{M}^{2}} ; \mathrm{K}=\frac{\pi f \mathrm{U}}{\mathrm{U}}
\end{aligned}
$$

Where, $\mathrm{K}$ - convective wave number, $\mathrm{M}$ - Mach number, $\mathrm{D}_{\mathrm{L}}$ is the low frequency directivity function, $\mathrm{f}-$ octave band frequency, Hz, LFC - Low frequency correction factor term given by (5), $l$ is the length scale, L - span segment length, $\mathrm{m}, \mathrm{I}-$ Turbulence intensity, \%, c - stream wise chord length, $\mathrm{m}, \mathrm{r}_{\mathrm{e}}$ is the distance between the non-stationary source and receiver, $\mathrm{m}, \mathrm{u}$ - mean wind speed along the rotor axis, $\mathrm{U}$ - relative velocity over the blade airfoil span, $\mathrm{S}$ is the compressible Sears function .

\subsection{Directivity formulation}

The directivity functions, $\mathrm{D}$, in the previous section explains about the directional nature of sound propagation in atmosphere which does not include the effects of refraction, edge scattering phenomenon, The trailing edge noise model by Amiet [1] considers the both low and high frequency directivity patterns from the source in far field conditions. The position of noise source is determined using the correction factors in shifted coordinate system and based on observer position relative to trailing or leading edge of airfoil.[5,18] The source convects shifts in direction of mean wind speed at 0.8 times the wind speed, that corresponds the atmospheric wind shear. All noise sources are assumed to originate from trailing edge except for inflow turbulent noise mechanism.

$$
\begin{aligned}
& \mathrm{D}_{\mathrm{H}}(\theta, \phi)=\frac{2 \sin ^{2}\left(\frac{1}{2} \theta\right) \sin ^{2}(\phi)}{(1+\mathrm{M} \cos \theta)\left(1+\left(\mathrm{M}-\mathrm{M}_{\mathrm{L}}\right) \cos \theta\right)^{2}} \\
& \mathrm{D}_{\mathrm{L}}(\theta, \phi)=\frac{\sin ^{2}(\theta) \sin ^{2}(\phi)}{(1+\mathrm{M} \cos \theta)^{4}}
\end{aligned}
$$

Where the term $\theta$ and $\varphi$ are the directivity angles between the observer and trailing edge of source in the span wise and chord wise directions. The term $1+M \cos \theta$ represents the Doppler shift and the convective amplification of source relative to observer.

\subsection{Integral length scale \& Turbulence intensity}

\section{Results And Discussion}

The velocity fluctuations in air cause turbulence around the blades. The blades of turbine encounter changing angle of attack that result in larger changes in lift and drag forces. The length scales and intensities are dependent upon the free stream velocity at site conditions and vary along the height above the ground. The isotropic turbulence in the atmosphere $[1,13]$ is function of hub height according to IEC standard and length scale are defined with respect to the rotor size. The size of length scale relative to leading edge radius of airfoil within the neutral stable boundary layer signifies that inflow noise behavior is a dipole source with sixth power 
Mach number or quadruple noise source at high frequencies. The source i.e. blades of turbine are considered as point dipole located at hub height and the sound radiated from them have high acoustic wave number compared to the distance between the source and receiver. The frequency spectra are evaluated using the scaling factor function which establishes the relationship between the convective wave number, $\mathrm{K}$ shed from the blade. Low frequency correction factor which takes account of compressible flow characteristic is expressed in terms of Mach number and compressible Sears function as proposed by Lowson [6].

Fig1. illustrates the effect of integral length scale, which is taken for $0.01 \mathrm{~m}, 1 \mathrm{~m}, 10 \mathrm{~m}$ and $35 \mathrm{~m}$, at constant turbulence intensity level i.e. $15 \%$, the graph of SPL $(\mathrm{dB})$ and octave center frequency ranging from 10 to $10^{5}$, which is the audible range that affects the perception of human being. The result shows that the SPL of $0.01 \mathrm{~m}$ length gives the least sound pressure level and increase in length scale produces more SPL (dB) indicating the sensitivity of acoustic pressure with length scales in size of rotor diameter. Further, at higher center frequency range the magnitude of SPL is observed to reduce. With increase in turbulence intensity, a similar trend can be noticed however, at lower center frequency the magnitude of SPL (dB) is quite large compared with higher frequency. At center frequency above $10^{4}$ the SPL (dB) levels continue to decrease. It must be noted that results from the trailing edge interaction noise of $3 \mathrm{MW}$ turbine on suction side is higher at low frequencies while the pressure side noise radiation is higher value of SPL $(\mathrm{dB})$ at higher frequencies. The angle dependent noise is high within narrow range in frequency spectra. The effects of inflow turbulent noise dominate the frequency spectra comparing to trailing edge interaction noise which is fairly large at lower center frequency band.
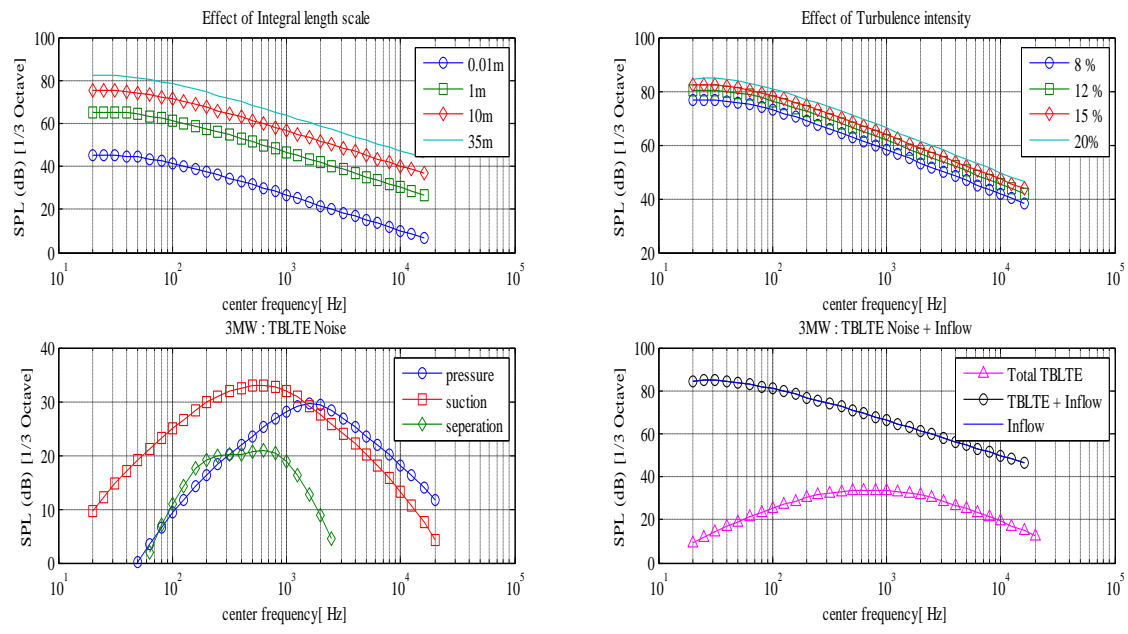

Figure 1 Turbulence inflow noise and Influence of length scales of 3MW turbine

\subsection{Mach number \& Rotor RPM}

The Mach number is a dimensionless quantity which signifies the flow velocity past a boundary relative to speed of sound. From fig 2 and fig 3 it can be inferred that as the rotor RPM of turbine is increased the relative velocity of blade particularly at tip, increase which result in higher Mach number along the blade span. The sound pressure levels SPL $(\mathrm{dB})$ are found to increase with higher rotational speeds of turbine. The maximum Mach number reached is $\sim 0.18$ near the tip of the blade $(90 \% \mathrm{R})$ while towards the root $(10 \% \mathrm{R})$ of the blade it is found to be $\sim 0.03$. Further, at $50 \% \mathrm{R}$, when the blade pass the tower, at 180 deg rotor azimuth the Mach number is found to reduce as result of relative velocity deficit due to tower wake for downwind turbines while for upwind machines the effect is observed lower. This trend is observed to be similar for all the turbines regardless of the size.

\subsection{Effects of power generation on SPL}

Fig 4 compares the sound pressure level from inflow turbulence with modified blade geometry configurations for $2 \mathrm{MW}$ turbine and power produced from the machine. For below rated wind speed the power production is affected, order of $22.5 \%$ lesser at wind speed of $8 \mathrm{~m} / \mathrm{s}$. This entails a decrease in SPL level assuming the rotor RPM, the environmental parameters such as the length scale and turbulence intensity are kept constant. The overall decrease in SPL $(\mathrm{dB})$ for the A weighted spectra is found to be $16 \mathrm{~dB}$ for observer azimuth position located at $30 \mathrm{deg}$. It can be inferred that increase (10\%) in chord length accompanied with decrease in twist $(5 \%)$ tend to reduce the SPL $(\mathrm{dB})$ levels by $16 \mathrm{~dB}$ considering the effects of noise from the turbulent inflow component only. The combined effect of noise from blade trailing edge interactions is logarithmically added with inflow noise is shown in Fig 1 (right). The quantitative comparison of A weighted acoustic spectra for all turbines is shown in fig. 3 and found to be high for $3 \mathrm{MW}$ machine 

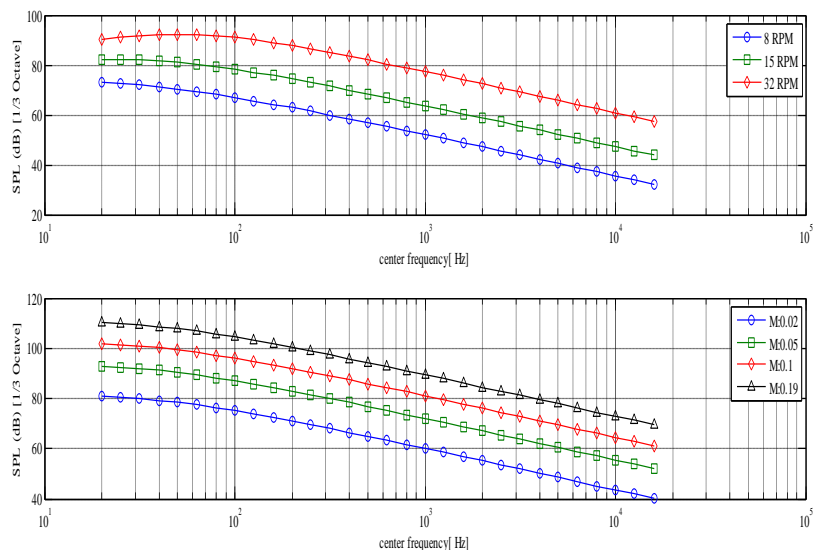

Figure 2 Inflow turbulence noise levels at Mach numbers \& Rotor RPM of 3MW machine.
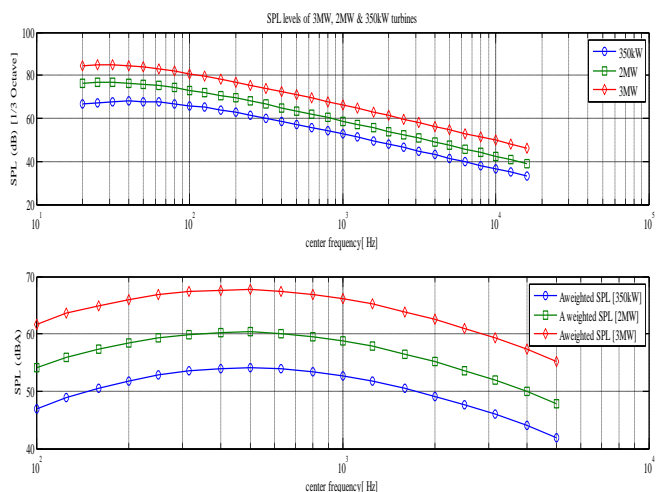

Figure 3 Comparison of sound pressure levels of 3MW, 2MW \& 350kW turbines
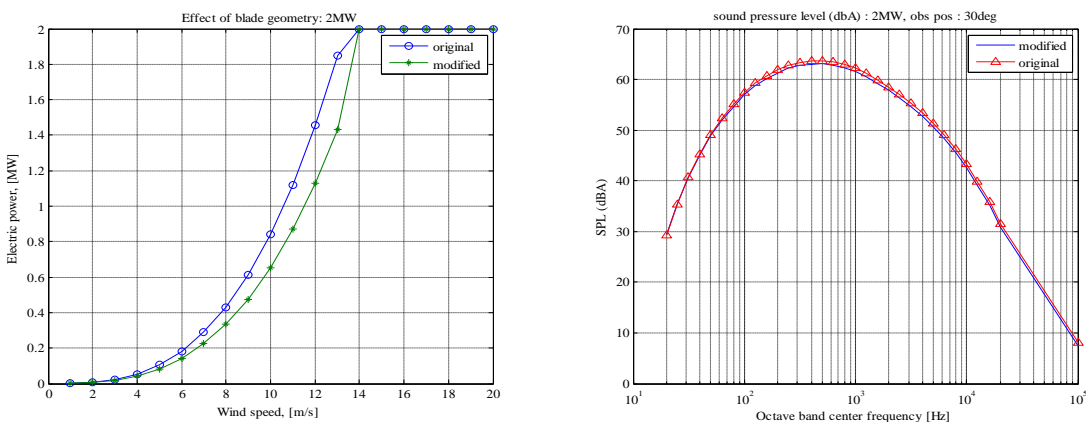

Figure 4 Effect of blade geometry on change in sound pressure level $(\mathrm{dB}) \&$ power production

\subsection{Amplitude modulation \& blade geometry}

Fig 5 illustrates the amplitude modulation at different octave frequencies ranging from $50 \mathrm{~Hz}, 100 \mathrm{~Hz}$, $200 \mathrm{~Hz}$ and $500 \mathrm{~Hz}$. The amplitude of $\sim 13 \mathrm{~dB}$, at $50 \mathrm{~Hz}$ is found maximum at $90^{\circ}$ observer azimuth angle and at $270^{\circ}$ observer azimuth angle in cross wind direction. The "swish" phenomenon is observed at frequencies 300 $\mathrm{Hz}$ to $5 \mathrm{kHz}[11,17]$. The plot depicts that as the octave frequencies band increases from 50 to $500 \mathrm{~Hz}$, the swish amplitude decreases for higher frequencies, 4-6 dB. However, the trend continues to remain the same for all octave band frequencies which is lower in upwind and downwind directions and higher in cross wind directions. This behavior is in contrast to the high and low frequency directivity pattern $[3,5,21]$ observed where sound pressure levels are lower in cross wind receiver positions. It must be noted that receiver heights and distance between the source and receiver are kept constant throughout for qualitative reasons. The "swish" amplitude or the modulation depth can be expressed mathematically using

$$
\Delta \mathrm{L}=20 \log \frac{\mathrm{p}_{\mathrm{raf}} \mathrm{f}^{2} \mathrm{pff}_{\mathrm{f}}}{\mathrm{pref}^{-2 \mathrm{p} f_{\mathrm{f}}}}
$$


Where, $p_{\text {ref }}$ is reference sound pressure $10^{-12} \sim 94 \mathrm{~dB}$ and $\mathrm{p}_{\mathrm{f}}$ is the directional sound pressure level $\mathrm{dB}$, The fig 5 depicts the comparison of the theoretical and numerical results with inflow and without inflow noise levels. With inflow the numerical results of sound power level (SPL) are found to be in good agreement with theoretical or analytical results of SPL for all the turbines. Further, the "swish" effect of turbine blades is often encountered higher in upwind direction than downwind when the blades reach horizontal position when moving downwards in the rotor plane. Noise radiated from "swish" is propagated and heard in different directions $[5,10]$ and depends upon observer position relative to turbine and no inherent change in magnitude are observed as blades rotate.This type of noise appears to originate from the trailing edge of the blades, and found usually in high frequency range of acoustic spectra in the near field. The magnitude of swish levels [5] perceived by receiver tend to prevail more during the night when the background noise levels. The blades also produce "thump" noise due to inflow turbulent velocity fluctuations prevalent in atmosphere. This type of noise, i.e. infrasound is dependent upon the leading edge thickness of the blades and roundedness which interact with the turbulent fluctuations of the wind and perceived higher in downwind direction when the blades cross the tower.
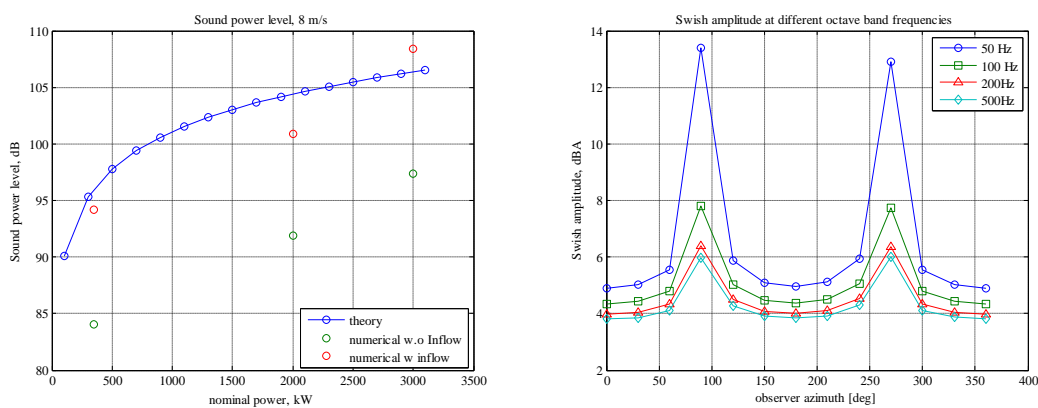

Figure 5 Swish amplitude \&Theoretical vs numerical comparison of Sound power levels

Further research by Bowdler [12] shows that amplitude modulation is caused due to a combination of directivity pattern emanating from trailing edge noise from blade and convective acoustic wave number, $\mathrm{K}$, due to the blade movement [5]. The modulation of blade noise is affected by the reflective surfaces close to wind turbine. Hard and reflective surface roughness increases the modulation depth [10, 11, 22]. Fig 5 illustrates modulation depth of $+/-4 \mathrm{~dB}$ (peak to trough) at distance of $80 \mathrm{~m}$ from turbine. The varying amplitude (increase or decrease) at different frequencies is subjected to synchronization effect when turbine is located in large array of wind turbines at which noise radiated from blades are propagated like waves and come in phase and out of phase. From previous studies [19,20] it is mentioned that perception of modulation is influenced by the propagation effects resulting from atmospheric factors such as refraction and due to acoustic edge scattering, obstacles, diffraction. Complete analysis of "swish" effect from blades is beyond the scope of study presented in this paper. Fig 6 (left) depicts the variation of Mach number with respect to rotor blade azimuth for three blades wind turbine. It is observed that at $0^{0}$ azimuth blade 2 experience maximum Mach number and the blade 3 has the minimum Mach number near the tip of blade. Fig 6 (right) depicts the variation of Mach number with respect to span length (length from root to tip measured as radius from root). Wind turbine design with aero acoustics viewpoint is governed by the tip speed, $\mathrm{m} / \mathrm{s}$ and rotor size of turbine. As the length of blade becomes large, the tip speed also increases and therefore noise levels produced from the turbine. At 10\% of radius (R), the Mach number is least and at $95 \%$ of radius the Mach number is highest. The inflow noise magnitude is insignificant for low Mach number flows compared to trailing edge noise [9, 15, 21] at high frequency. Fig 7 shows Mach number variation along the blade span, rotor azimuth angles for 3MW, $2 \mathrm{MW} \& 0.35 \mathrm{MW}$ machines calculated at $8 \mathrm{~m} / \mathrm{s}$.
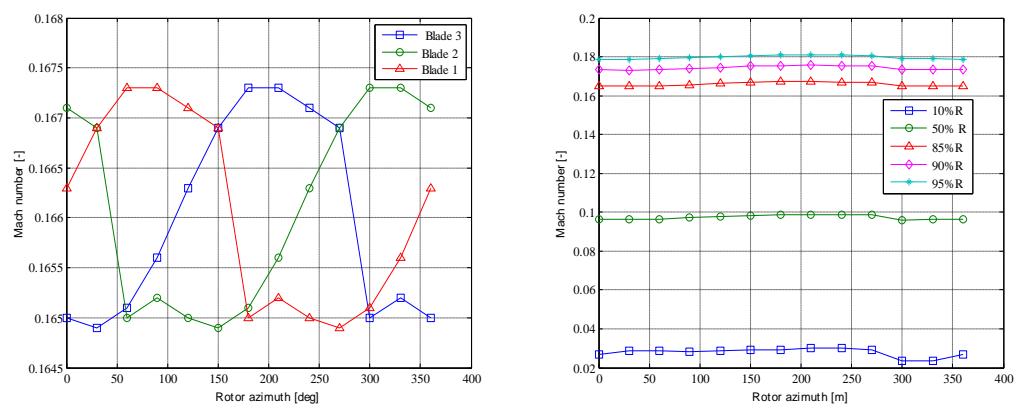

Figure 6 Mach number variations along Rotor azimuth for individual blades and span stations 

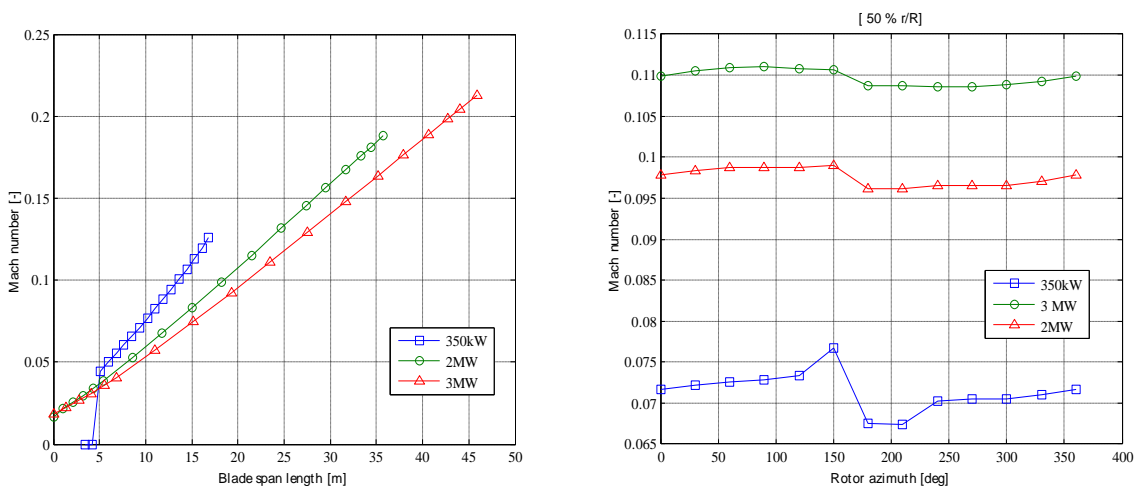

Figure 7 Mach number along blade span $[\mathrm{m}]$ for $350 \mathrm{~kW}$, 2MW \& 3MW turbines at $8 \mathrm{~m} / \mathrm{s}$
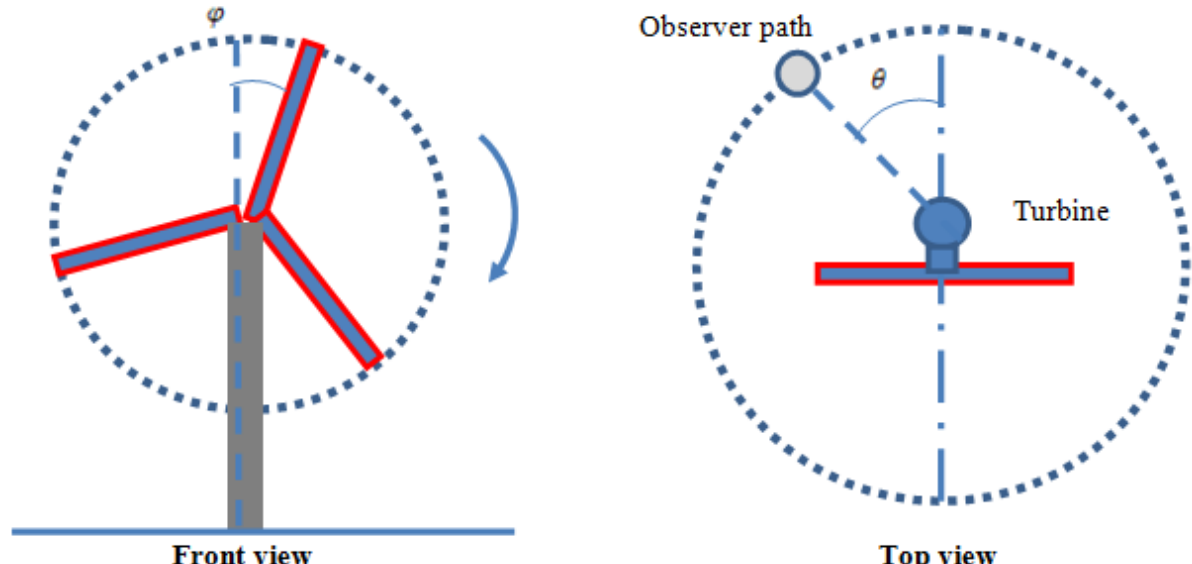

Top view

Figure 8 Schematic diagrams of Rotor azimuth and Observer azimuth angles, $\psi$ and $\theta[23]$

For a wind turbine, when the blades move past the tower, the reduction in relative velocity occurs due to presence of tower. The tower wake causes blade loading in the rotor plane for downwind turbines. For upwind turbines, the velocity deficit upstream causes reduction in power production which can be observed when the blades reach bottom of rotor plane in front of tower, i.e. $180 \mathrm{deg}$. The result effects of tower wake and wind shear effect on the power production from a $2 \mathrm{MW}$ turbine configuration is assessed. The tower effect [8] is modeled in BEM code and calculated for wind speeds range of $20 \mathrm{~m} / \mathrm{s}$. The shear effect $[5,6,8]$, is also modeled in BEM using the surface roughness parameter at the site location.

The power curve of turbine considers wind speeds upto $25 \mathrm{~m} / \mathrm{s}$, however, the BEM computations are done for wind speeds of $20 \mathrm{~m} / \mathrm{s}$ as the turbines are pitch regulated variable speed type. The rated power of machine is found between $11-13 \mathrm{~m} / \mathrm{s}$ for a rotor diameter of $75 \mathrm{~m}$ as shown in fig 4 . fig 9 shows the tower effect for the three blades when they pass the tower at rotor azimuth positions of $60 \mathrm{deg}, 180 \mathrm{deg}$ and $300 \mathrm{deg}$ for different wind speeds $6 \mathrm{~m} / \mathrm{s}, 8 \mathrm{~m} / \mathrm{s}$ and $9 \mathrm{~m} / \mathrm{s}$ respectively. Further, the wind shear effect is computed with an increment of $100 \%$ in the shear exponent values. The difference in magnitude of power production is plotted at wind speed of $9 \mathrm{~m} / \mathrm{s}$ for wind shear of 0.2 and 0.1 . The velocity gradient above ground can be calculated using $\mathrm{V}=\mathrm{V}_{0}\left(\frac{\mathrm{z}}{\mathrm{z}_{0}}\right)^{\mathrm{x}}$

Where $\mathrm{z}$ - surface roughness at height, $\mathrm{h}$, above ground; $\mathrm{z}_{0}$ is surface roughness at ground reference height usually $10 \mathrm{~m} . \mathrm{V}_{\mathrm{o}}$ is the reference wind speed measured at the monitoring met mast, $\mathrm{V}$ is the velocity approximation at the desired height, $\mathrm{h}$ above the ground. $\alpha$ is measure of wind shear exponent at the site. 

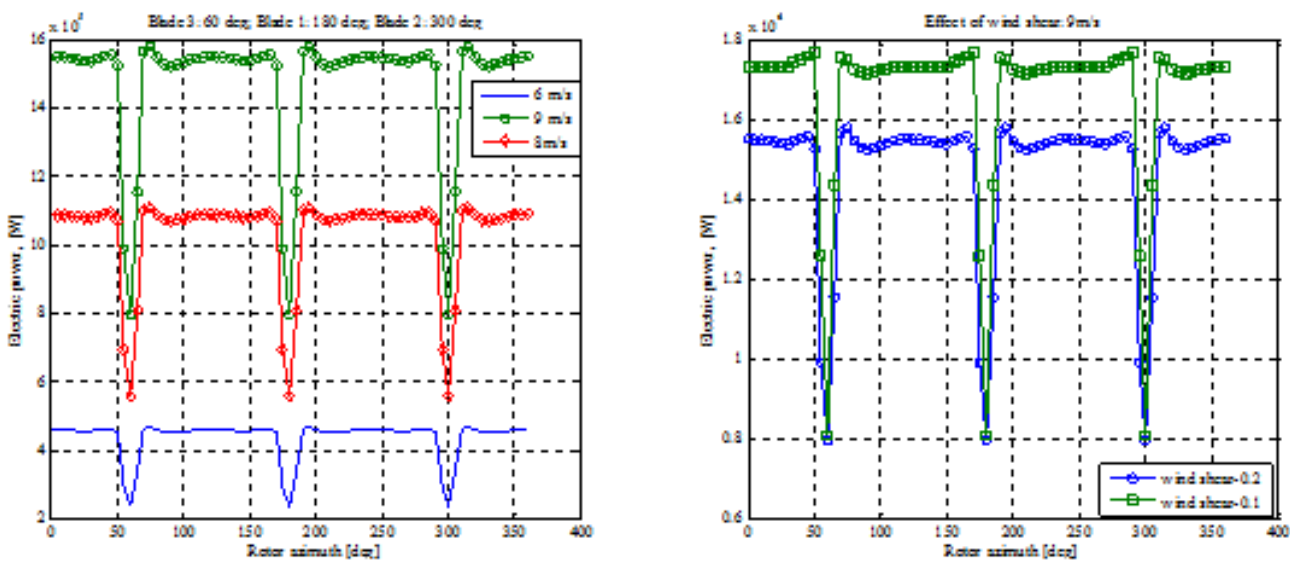

Figure 9 Tower effect \& wind shear on power production: 2MW turbine

Fig.10 shows the comparison of relative velocity of blade at different rotor azimuth angles without yawing as well for the yaw angle of $15 \mathrm{deg}$. During the yawing operation of turbine, the blade velocity is found to be reduced when the blade reaches the top position than when the blade is at bottom. It can be noted that for power production during yaw operation is higher compared when the turbine is not yawing, The SPL (dB) levels for different observer azimuth angle are plotted in fig. 9 which indicates that at $90 \mathrm{deg}$ in cross wind direction, the sound pressure level is lower compared to $0 \mathrm{deg} / 180 \mathrm{deg}$ downwind /upwind directions. The tower wake produces rotor in-plane velocity gradients and it also causes the impulse noise to be produced in the form of acoustic pulses [6,7]. The pulses are transient in nature and vary in amplitude. Such type of noise source is predominant in downwind turbines and can be eliminated using upwind configuration by placing the rotor ahead of tower. The impulse noise can be expressed in terms of Fourier components at blade passing frequency and integer harmonics of it.[16,17,19]
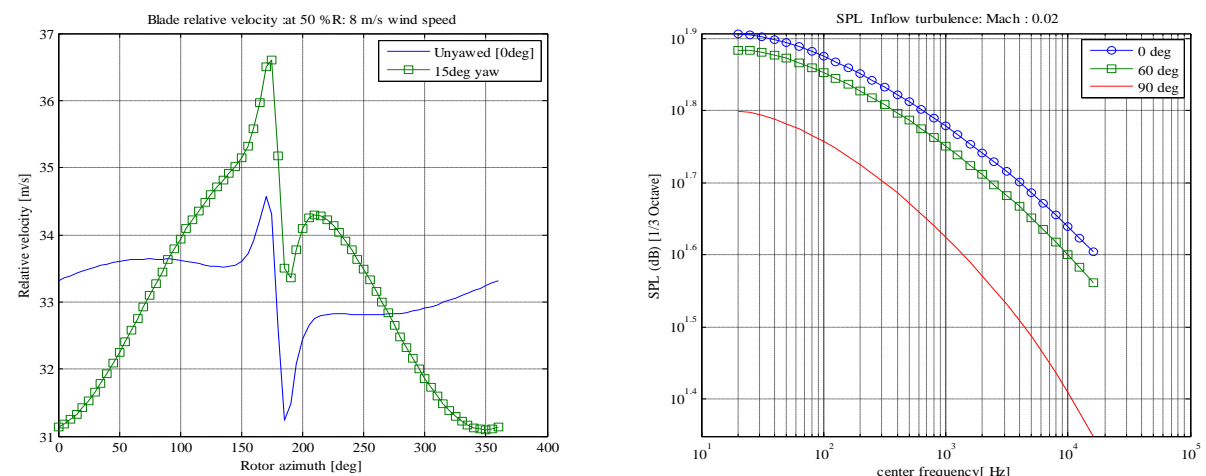

Figure 10 Blade relative velocity during yaw\& frequency spectra SPL (dB) with observer azimuth [deg]

\section{Conclusions}

Turbulent inflow noise is predominantly a low frequency phenomenon dependent upon the atmospheric length scales and turbulent intensity levels in acoustic spectrum. The magnitudes of inflow noise mechanism dominate in overall sound pressure level produced from turbine. The length scales factor is sensitive to the rotor size of turbine. The rotational noise is governed by blade tip speed and rotor diameter. With increase in Mach number and rotor RPM, the sound pressure levels are found to increase by order of $10 \%$. The noise radiated increase by $2 \mathrm{~dB}$ for every $1 \%$ increase in turbulence intensity while 8-15 dB increased with higher length scale. The blade twist and chord have pronounced effect on sound pressure levels and power production from turbine.

The observer azimuth angle is key factor in sound pressure level perceived by receiver and found maximum in downwind and upwind directions at high frequencies and attenuate due to refraction effects and surround obstacles. The "swish" amplitudes from blade vary with observer position and found higher in upwind than in downwind direction. The amplitude modulation of wind turbine noise is perceived clearly in the near field compared to far field. The velocity deficit caused due to tower wake affect the power produced from turbine and generate the impulse noise at blade passing frequency of turbine. The blade and tower wake interactions are complex in nature and noise produced due to them can be eliminated in upwind turbine configurations. 


\section{Nomenclature}

SPL - Sound Pressure Level, Sound Power Level

$\mathrm{dB}$ - Decibel

$\mathrm{dBA}$ - A weighted sound pressure level

BPM - Brooks, Pope, Marcolini

BEM - Boundary Element Momentum

RPM - Rotations per Minute

MW - Mega Watt

kW - Kilo Watt

LFC - Low Frequency Correction

$\mathrm{TI}$ - Turbulence Intensity

\section{References}

[1]. R.K Amiet. Noise due to turbulent flow past a trailing edge. Journal of Sound and Vibration. 47(3) 387-393. (1976)

[2]. R.K. Amiet. High-frequency thin airfoil theory for subsonic flow. AIAA J., 14(8), 1076\{1082, (1976).

[3]. M. S. Howe. The influence of vortex shedding on the generation of sound by convected turbulence. J. Fluid Mech., 76(4), 711\{740, (1976).

[4]. Richard D. Sandberga, Neil D. Sandham Noise due to unsteady flow past trailing edges University of Southampton, Aerodynamics and Flight Mechanics Research Group, U.K.

[5]. P. Moriarty, P. Migliore. Semi empirical aero-acoustic Noise prediction code for Wind turbines. Technical Report, NREL. December 2003. NREL /TP-500-34478.

[6]. Harvey Hubbard, Kevin P Shepherd. Aeroacoustics of large wind turbines. NASA Langley Research center, Hampton, Virginia.

[7]. F.W Grosveld, Broadband noise prediction from horizontal axis wind turbines, Bio-mimetics Corporation Hampton, Virginia

[8]. M. Hansen. Unsteady BEM simulation, DTU, Kongens, Lyngby, Denmark.

[9]. Seunghoon Lee, Seungmin Lee, Soogabh Lee. Numerical prediction of acoustic pressure radiated from large wind turbines. Inter noise 2011Osaka Japan, Sep 4-7. Aero-acoustics and Noise control laboratory. Seoul National University,Seoul.

[10]. Hogeon Kim, Soogab Lee. Aerodynamic noise analysis of large horizontal axis wind turbines considering fluid structure interaction. Seoul National University, Republic of Korea.

[11]. Rufin Makarewicz, Roman Golebiewiski, Amplitude modulation of wind turbine noise. Institute of Acoustics, A Mickiewicz University, Poland.

[12]. Dick Bowdler, Amplitude modulation of wind turbine noise, A review of evidence. New Acoustics, UK

[13]. Bharat Raj Agrawal, Aaron Rosenberg, Anupam Sharma, Towards identifying contribution of wake turbulence to Inflow noise from wind turbines Iowa state university, Iowa, USA.

[14]. Oriol Ferret Gasch, Assessment, development and validation of wind turbine rotor noise prediction codes, June 2014, DTU Wind energy, Lyngby, Denmark.

[15]. Valeri V Lenchine, Amplitude modulation in Wind turbine noise. Science and sustainability division, EPA Adelaide, Australia.

[16]. Peter Djikstra, Rotor noise and aero acoustic optimization of wind turbine airfoils. TU Delft. The Netherlands.

[17]. Moller Henrik, Pedersen, Christian Sejer Low frequency noise from large wind turbines. 2011, Aalborg university, Denmark.

[18]. Manne Friman, Directivity of sound from wind turbines, A study of horizontal radiation pattern from wind turbine, Department of Aeronautical \& Vehicle engineering, KTH, Stockholm ,Sweden.

[19]. Henrik Moller, Pedersen, Christian, Low frequency noise from large wind turbines," Aalborg University, Denmark.

[20]. C Doolan, Danielle Moreau, Cristobal Albarracin, Trailing edge noise production, prediction and control, School of mechanical engineering, University of Adelaide, South Australia, 5005, Australia.

[21]. W.Zhu, J. Sorensen, W. Shen, Modeling of noise from wind turbines, Technical University of Denmark, Lyngby, Denmark.

[22]. Mathew Cand et al. Wind turbine amplitude modulation: Cause and effect. Proceedings of Acoustics 2012, Nantes Conference.

[23]. IEC 61400-11, Wind turbine generator systems, Acoustic noise measurement techniques (2002). 